\title{
APPRENDERE L'ITALIANO OGGI
}

PAOLA MicheLi

ABSTRACT L'apprendimento dell'italiano all'estero e in Italia: comè̀ cambiato il pubblico e le sue motivazioni. Il fenomeno dell'immigrazione in Italia ha aperto nuove prospettive nel processo di insegnamentoapprendimento.

PAROLE CHIAVE apprendimento; motivazioni; immigrazione; insegnamento.

RFST:MO A aprendizagem do italiano no exterior e na Itália: como mudou o público e suas motivações. O fenômeno da imigração na Itália abriu novas perspectivas ao processo de ensino/aprendizagem.

I:HLAVRA-CHAV! aprendizagem; motivações; imigração; ensino.

ABSI'LAC'T The learning of italian language in Italy and abroad: how the students and theirs incentives have changed. New perspectives in the teaching-learning process are opened in Italy by event of immigration.

KETWO LS learning; incentives; immigration; teaching. 


\section{Le motivazioni allo studio della lingua italiana all'estero}

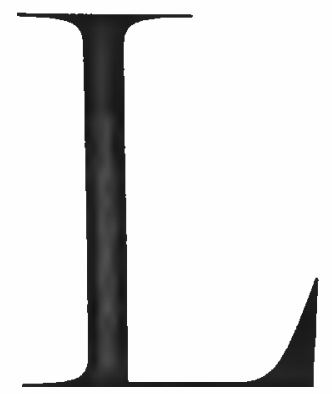

a più recente indagine sui pubblici e sulle motivazioni dell'italiano nel mondo è Italiano 2000, promossa dal Ministero degli Affari Esteri, affidata al Dipartimento di Studi Linguistici e Letterari dell'Università di Roma "La Sapienza" e realizzata sotto la direzione di Tullio De Mauro, in collaborazione con Massimo Vedovelli, Monica Barni e Lorenzo Miraglia dell'Università per Stranieri di Siena.

Italiano 2000 ha avuto l'obiettivo di raccogliere dati sullo stato attuale della lingua italiana nel mondo e sugli interventi per la sua diffusione, con particolare riferimento alle caratteristiche, alle motivazioni e ai bisogni formativi dei pubblici stranieri dell'italiano; ai tratti metodologici dell'offerta formativa; al quadro generale delle condizioni che determinano la posizione e lo sviluppo della nostra lingua nel mondo. ${ }^{1}$ (De Mauro, Vedovelli, Barni, Miraglia: 2002)

La materia di questa ricerca fa riferimento ad altre precedenti indagini, come quella svolta alla fine degli anni '70 dall'Istituto dell'Enciclopedia Italiana, già per conto del M.A.E., sotto la direzione di Ignazio Baldelli, e anche a quella promossa più recentemente dalla Società Dante Alighieri, allo scopo di fornire dati aggiornati e sistematici sull'italiano nel mondo.

Il dato quantitativo comune che emerge dalle indagini, anche di quelle che hanno preceduto in ordine temporale Italiano 2000 , è che nell'arco di circa un ventennio l'espansione dello studio della lingua italiana è in crescita costante, malgrado si sia attestato, a metà Novecento, su posizioni di notevole distacco rispetto allo studio di altre lingue. Oggi questo distacco si sta gradualmente 
colmando, tanto che l'italiano è attestato nelle prime posizioni fra le lingue europee tradizionalmente più richieste (francese, spagnolo, tedesco) o anche fra lingue come il giapponese, il russo, l'arabo. Al di là dei dati quantitativi, l'indagine ha prodotto dati di tipo qualitativo di grande interesse, avendo tra i presupposti principali quello di fornire informazioni il più possibile dettagliate sulla composizione dei pubblici di italiano nel mondo e sulle motivazioni allo studio.

La convinzione, ancora molto forte in anni recenti, che lo studio dell'italiano fosse all'estero solo prerogativa dei ceti più colti e quindi rappresentasse un forte richiamo dovuto alla cultura alta italiana da parte di studiosi e cultori delle arti e della musica, viene superata dai dati ricavati da questa indagine, che tuttavia risultano assai articolati. Accanto infatti a esigenze di studio e di ricerca, che pur sopravvivono, tra le motivazioni salienti per la scelta dell'italiano si annoverano esigenze di lavoro (circa un quarto degli apprendenti) presso imprese industriali e commerciali come anche il bisogno di stabilire relazioni sociali o di tipo turistico, senza dimenticare che anche questo settore non presenta oramai più il carattere elitario che aveva una volta.

La dimensione economica costituisce dunque una delle motivazioni più forti allo studio dell'italiano e che fa si che l'italiano occupi una posizione di rilievo nel «mercato delle lingue», ${ }^{2}$ vale a dire in quella dimensione secondo la quale la diffusione di una lingua a livello internazionale non è solo

il frutto della intrinseca identità semiotica e strutturale delle lingue, ma anche del sistema che nella lingua fa convergere le dinamiche sociali e produttive delle comunità dei suoi utenti primari, le politiche istituzionali di produzione della sua diffusione, la capacità che le società hanno di proporre al resto del sistema internazionale le proprie identità sociali e culturali in termini di punti di riferimento, di modelli dotati di prestigio e successo. (Vedovelli, 2005a)

Ciò significa che la diffusione delle lingue sul piano internazionale è determinata dagli elementi linguistici, ma anche dalla capacità di una società a porsi a livello internazionale come sistema sociale capace di ricevere attenzione. La capacità di attrazione di una lingua assume dunque un rilievo determinante insieme al sistema sociale, economico e culturale di cui è veicolo.

E infatti la presenza vitale della lingua italiana nel mercato delle lingue è in stretta relazione con i valori culturali e con una più generale concezione di buon gusto. Basti pensare alla massiccia presenza di parole italiane che a livello internazionale vengono usate in tutte quelle manifestazioni produttive che hanno fatto si che l'Italia occupasse i primi posti tra i paesi più industrializzati del mondo e, nei settori come la moda, la gastronomia, il design, puntualmente associate a una dimensione estetica e creativa.

2. Dallo studio di L.-J. Calvet Le marché aux langues, Paris, Plon, 2000, si estrae il concetto di "mercato delle lingue" in riferimento alla competitività tra le lingue nel più vasto panorama mondiaie. 


\section{Lo studio della lingua italiana in Italia}

La molteplicità dei pubblici e la varietà delle motivazioni all'apprendimento dell'italiano portano a differenziare i presupposti che stanno alla base del processo di insegnamento-apprendimento della nostra lingua. Tale fenomeno non si verifica nel caso di una lingua come l'inglese che, grazie al carattere meramente strumentale della comunicazione che sta alla base dell'apprendimento, presenta una maggiore standardizzazione dei processi di formazione linguistica. I termini più ricorrenti usati in riferimento alla condizione della lingua italiana sono prevalentemente quelli di "Lingua straniera" (LS), "Lingua seconda" (L2) e "Lingua etnica” per definire rispettivamente la lingua italiana come oggetto di apprendimento all'estero, oggetto di acquisizione in Italia, lingua delle comunità italiane emigrate all'estero.

Recentemente siè diffuso tragli specialisti ancheil termine "lingua di contatto", usato principalmente per delineare la condizione dei figli degli immigrati stranieri in Italia per i quali l'italiano non è definibile né come L1 né come L2. La competenza comunicativa di questi alunni è infatti il luogo di contatto tra più codici linguistici e culturali che creano nuove identità, ma nel loro complesso contribuiscono a creare un'identità al confine fra la lingua materna e la L2.

L'espressione "italiano lingua di contatto" è stata usata ufficialmente per la prima volta in un documento programmatico della Commissione ministeriale per il riordino dei cicli scolastici, nominata nel 2000 da Tullio De Mauro, allora Ministro della Pubblica Istruzione, proprio per riferirsi agli alunni stranieri immigrati in Italia. Non è infatti un dato trascurabile il fatto che proprio entro i confini nazionali risieda una parte consistente del pubblico straniero dell'italiano: circa due milioni e mezzo di immigrati da altri paesi sono coinvolti in un processo di apprendimento della competenza linguistico-comunicativa, a livello spontaneo o in contesto formale, prevalentemente nei Centri Territoriali Permanenti per l'educazione degli adulti. All'interno della realtà che vede ormai l'Italia come paese di immigrazione, è proprio per le nuove generazioni di origine straniera che l'italiano contribuisce a creare un ambiente di contatto all'interno del quale ogni individuo ridefinisce la propria identità prima di tutto linguistica.

Il primo saggio di carattere sociologico sul fenomeno dell'immigrazione in Italia, allora fenomeno del tutto nuovo, risale al 1979; mentre il primo articolo sui problemi linguistici degli immigrati stranieri è del $1981 .{ }^{3}$ Oggi, come già accennato, l'italiano costituisce la lingua di contatto per circa due milioni e mezzo di immigrati stranieri adulti e bambini, per i quali la motivazione prevalente non è tanto quella dell'interesse culturale né tantomeno del turismo, ma piuttosto del vivere e sopravvivere in Italia allo scopo di inserirsi socialmente e professionalmente.

Se l'indagine Italiano 2000 ha contribuito a fornire dati di assoluta rilevanza sull'italiano diffuso fra gli stranieri, soprattutto fuori dai confini nazionali, per quanto riguarda la nostra lingua diffusa fra gli immigrati stranieri attualmente presenti in Italia, è possibile fare riferimento alle indagini conoscitive del Dossier Caritas (a partire dal 2001) e del Centro di eccellenza della ricerca

3. Esperienze e Proposte, 1979, Documentazione di base per una indagine sui lavoratori stranieri in Italia, Cattedra di Sociologia 2B, Università di Roma, "Esperienze e proposte", 38, 1979.

Massimo Vedovelli, 1981, La lingua degli stranieri in Italia. "Lingua e Nuova Didattíca", 3, 10:17-23. 
Osservatorio linguistico permanente dell'italiano diffuso fra stranieri e delle lingue immigrate in Italia, costituito dal Ministero dell'Università presso l'Università per Stranieri di Siena e diretto dal Prof. Massimo Vedovelli.

Lapercentuale degli immigrati che studiano l'italiano in un contesto guidato è oggi in aumento. Tra questi possiamo intravedere due diverse situazioni: gli adulti che hanno superato l'età dell'obbligo scolastico in Italia e i giovani (bambini e adolescenti) che sono inseriti nei diversi gradi di istruzione del sistema scolastico italiano (scuole materne, elementari, medie e superiori). I primi immigrati con i quali gli insegnanti di italiano si trovarono a operare erano adulti di età compresa tra venti e quarant'anni, prevalentemente maschi. I materiali didattici e le strutture a disposizione si rivelarono da subito insufficienti e la preparazione degli insegnanti del tutto inadeguata, mancando all'epoca una tradizione di insegnamento per questo tipo di pubblico.

Oggi la formazione linguistica degli immigrati adultiè gestita principalmente dai Centri Territoriali Permanenti per la formazione degli adulti (CTP), strutture territoriali istituite dal Ministero per la Pubblica Istruzione nel 1997. Interessante è l'esperienza toscana che, nell'ambito di una nascente rete dei CTP, ha favorito lo sviluppo di linee comuni per l'insegnamento dell'italiano L2, con l'intenzione di veicolare le migliori esperienze dei singoli Centri e di fornire un punto di riferimento per tutti quegli operatori impegnati in aspetti progettuali, organizzativi e didattici. Da questa esperienza è nato il volume Italiano L2. Vademecum per gli operatori dei Centri Territoriali Permanenti. ${ }^{4}$ La pubblicazione propone l'approccio pragmatico e sociolinguistico del Quadro Comune Europeo di Riferimento (QCER) e ad esso è ispirato anche nella definizione dei profili di apprendenti e dei livelli di competenza linguistico-comunicativa: livello basico, livello indipendente, livello competente.

Un'esperienza decisamente innovativa è stata portata avanti dall'Università per Stranieri di Siena in collaborazione con il quotidiano 'La Repubblica' attraverso l'inserto 'Metropoli', Il giornale dell'Italia multietnica, (www.metropoli.repubblica.it). A partire dal settembre 2006 per undici settimane è stato pubblicato un corso di italiano in undici lezioni «per muoversi nella vita di tutti $i$ giorni, ma anche per acquistare quella piena padronanza dei costumi e delle leggi che è necessaria a ogni cittadino italiano. Il corso non è un testo di grammatica. Insegna le parole e il loro uso, ma anche il modo di muoversi in un paese diverso da quello di origine». É stato articolato con una parte generale e una parte più specifica dedicata alla formazione linguistica nei settori dell'assistenza familiare (come comunicare con gli anziani) o del fare acquisti (fare la spesa, scegliere cibi). La parte generale si rivolge agli immigrati appena arrivati o che si trovano da poco tempo in Italia e può essere utile sia per gli adulti che per i bambini e usata in una scuola o in famiglia, da soli o in gruppo. Ogni lezione è divisa in due parti: la prima, "Impariamo l'italiano", fornisce gli elementi di base della lingua e la seconda, "Testi e usi", presenta le situazioni più frequenti della vita quotidiana. Come si legge nell'introduzione, «il corso è anche un atto di amicizia, un modo di dimostrare ai lettori di "Metropoli" che non sono soli in Italia e che siamo accanto a loro per aiutarli».5

4. Borgogni M.C., Giannini C., Leonardis L., Piazzesi S., Pucci M., 2004, Firenze, Rete Toscana dei Centri 'Territoriali Permanenti - IRRE Toscana - Grafiche Cappelli.

5. Metropoli, anno 1, n.28. Hanno collaborato al corso di lingua Carla Bagna, Monica Barni, Alessandro Pallassini, Stefania Semplici, Donatella Troncarelli, Massimo Vedovelli (Unità 1-3) insieme anche a Sergio Pizziconi e Isabella Totaro (Unità 4-11). 
I giovani immigrati ancora in età scolare sono più presenti nelle scuole elementari e materne e in misura minore nelle scuole medie e superiori, malgrado anche qui si registri un progressivo aumento di presenze.

Mentre nei CTP gli immigrati adulti vedono rispondere in maniera specifica alle loro esigenze, grazie a insegnanti formati e a strutture didattiche idonee, nei diversi cicli scolastici molto è stato fatto, ma restano ancora numerosi problemi da risolvere. Gli insegnanti si trovano spesso a dover fare i conti con allievi che, non conoscendo affatto la lingua, non sono in grado di interagire con l'ambiente che li circonda.

In particolare, proprio il fenomeno in crescita degli adolescenti presenti nelle scuole italiane merita attenzione per intraprendere uno studio circostanziato della loro situazione.

I presupposti da cui partire nel considerare la situazione linguistica degli adolescenti immigrati inseriti nella scuola superiore italiana riguardano innanzitutto le caratteristiche psico-evolutive, le modalità di apprendimento, i processi cognitivi e le esigenze che sono uniche per questa fascia di apprendenti e che meritano pertanto uno studio specifico, soprattutto in considerazione del fatto che se la condizione di migrante porta già di per sé una ridefinizione dell'identità dell'individuo che nasce dal contatto con una nuova lingua e cultura, il fatto di vivere questa condizione in età adolescenziale fa si che a questa ristrutturazione dell'individuo si sommi anche il cambiamento e la ridefinizione dell'identità che normalmente si produce in ogni individuo in quella fascia di età, rendendo cosi ancora più complessa la gestione dei processi di definizione dell’identità. (Gallina, 2004)

Un quadro teorico di riferimento per questo studio può essere definito tenendo conto delle prospettive semiotiche, degli studi sul contatto linguistico, degli studi di linguistica acquisizionale e di glottodidattica. $\dot{E}$ quindi opportuno rifarsi, da una parte, agli studi che descrivono i processi di apprendimento attraverso le tappe dello sviluppo interlinguistico e, dall'altra, agli studi che definiscono la competenza a cui devono arrivare gli apprendenti per gestire l'interazione nei diversi scambi sociali e comunicativi per giungere a una didattica di tipo acquisizionale (Vedovelli, Villarini 2003) che tenga conto dello sviluppo della competenza linguistico-comunicativa degli apprendenti stranieri e individuare interventi didattici che tengano conto di questi processi, cercando di conciliare il piano teorico e descrittivo con quello applicativo-operativo e regolativo.

La scuola dell'obbligo rappresenta il primo contesto in cui si devono dare risposte adeguate alle esigenze di sviluppo sociale e culturale dell'immigrato straniero. Molto è stato fatto dopo un primo momento in cui si è cercato di adattare agli stranieri modelli formativi normalmente adottati con alunni italiani e successivamente avvalendosi di modelli specifici prevalentemente ispirati a paradigmi dell'intercultura. Parallelamente ci si è orientati su processi di cambiamento della professionalità 
dei docenti di italiano Ll in docenti di italiano L2 attraverso l'organizzazione di corsi di formazione, mentre le Università per Stranieri di Siena e Perugia hanno attivato corsi di diploma, di laurea e scuole di perfezionamento mirati a formare docenti di italiano L2. Tuttavia è ancora frequente l'inserimento, nella scuola dell'obbligo, di giovani alunni in classi inferiori alla loro età. A tale proposito è interessante lo studio, in fase sperimentale, di Eleonora Fragai (Università per Stranieri di Siena) che prevede di applicare ai giovani immigrati il progetto di educazione linguistica elaborato negli anni ' 80 da Tullio De Mauro e dal suo gruppo di lavoro, il glotto-kit, per conoscere i bisogni degli allievi e individuare gli specifici obiettivi e i percorsi di formazione. ${ }^{6}$ La sperimentazione è stata svolta a Cortona (Arezzo) somministrando a un piccolo gruppo di allievi stranieri tra i 6 e i 17 anni, inseriti nelle scuole dell'obbligo, questionari socioculturali e prove linguistiche. I dati ricavati, una volta sottoposti ad analisi statistiche, hanno confermato il ruolo rilevante di certi fattori socio-culturali sul processo di costruzione dell'identità linguistica di bambini e adolescenti stranieri inseriti nel contesto istituzionalizzato di apprendimento. Questo comporta sul piano operativo

l'esigenza di riflettere innanzitutto sui fenomeni di socializzazione in generale e sulla necessità di garantire agli allievi stranieri un inserimento entro un contesto di apprendimento ricco e stimolante, come presupposto fondamentale allo sviluppo della competenza linguistica e comunicativa in L2, dipendente innanzitutto da componenti motivazionali legate al desiderio di appartenenza nei confronti del gruppo verso cui è orientato l'atteggiamento di identificazione. (Fragai, 2000)

\section{Linee comuni per l'insegnamento dell'italiano L2}

Le recenti indicazioni di politica linguistica del Consiglio d'Europa contenute in un documento che oggi è uno strumento imprescindibile per quanti operano nell'insegnamento linguistico, il Common European Framework for Languages: Learning, Teaching, Assessment, noto in Italia con il nome di Quadro Comune Europeo di Riferimento (QCER), offrono per l'organizzazione e la didattica un contributo fondamentale per sviluppo di modalità condivisibili, creando un linguaggio comune fra tutti quanti sono coinvolti in attività di apprendimento e insegnamento linguistico. Chi usa e apprende una lingua è considerato un agente sociale che deve portare a termine determinati compiti linguistici «in circostanze date, in ambiente specifico e all'interno di un determinato campo di azione», attraverso «attività linguistiche» di ricezione, interazione e produzione di testi. Dunque il punto di partenza di ogni azione didattica è basato su una visione della lingua come uso all'interno di una dimensione sociale e interazionale. Il concetto di "competenza linguistico-comunicativa" prevede il superamento dell'opposizione "competenza linguistica/competenza comunicativa". Difatti lalingua

6.Una illustrazione dettagliata dello studio di Fragai è reperibile in 'Studi Emigrazione', anno XXXVII, 2000, n.140 pp.963-980. Ibidem una bibliografia ragionata di studi sull'italiano degli immigrati in Italia. Per una bibliografia di studi sulla lingua degli emigrati italiani all'estero si veda 'Studi Emigrazione', anno XXXV, 1998, n.132. 
è considerata un sistema di usi linguistici che la persona mette in atto in un dato contesto sociale e da questo sono condizionati. «L'utente/apprendente è un soggetto sociale, cioè ha un'identità che si stabilisce entro il gruppo sociale, e per svolgere i compiti sociali si relaziona agli altri; svolge questi compiti in un determinato contesto che definisce il loro senso" (Vedovelli, 2000:38).

La competenza in una lingua non si limita pertanto al "sapere" in termini di conoscenza delle forme e delle sue strutture, ma di "saper fare" di saper agire in determinati contesti di comunicazione; non è quindi separabile dalle capacità individuali e nemmeno dalle relazioni contestuali individuali e collettive, concretizzandosi nella processazione di testi, determinati anch'essi dal contesto dell'interazione e che costituiscono l'unità minima di comunicazione.

Nel QCER la competenza linguistico-comunicativa è presentata come un continuum articolato su vari piani e, a seconda del grado di mobilità dell'individuo al suo interno, è indice di progressione della competenza linguistica. Questa potenziale progressione si esplica nell'articolazione in livelli che, attraverso i vari descrittori, formalizzano i diversi gradi di apprendimento.

Come si è già detto, anche per l'apprendimento dell'italiano il QCER costituisce un importantissimo strumento se usato come quadro di riferimento; purtroppo è necessario prendere atto che proprio i descrittori di competenza costituiscono dei possibili rischi per un'utilizzazione impropria del QCER, se questi vengono recepiti come strumento prescrittivo, con ricadute molto dannose in ambito formativo. Ma è proprio in base all'articolazione in livelli tramite i vari descrittori che

è possibile sia definire un modello di obiettivi didattici coerente, trasparente e comprensibile a tutti, sia ogni operazione di valutazione e, in particolare, di tipo certificatorio, perché i descrittori di ciascun livello diventano il punto di riferimento, il criterio in base al quale verificare la competenza raggiunta: condizione necessaria per costruire prove adeguate e per misurare e valutare le prestazioni degli apprendenti sulla base di ciò che i contesti di uso linguistico richiedono. ${ }^{7}$

Proprio in stretto legame con le sollecitazioni provenienti dal QCER nascono le certificazioni di italiano. In particolare, le numerose e dettagliate indagini condotte in seno al Centro di certificazione CILS dell'Università per Stranieri di Siena hanno portato, oltre alla costruzione di sei moduli certificatori, alla realizzazione e sperimentazione di moduli differenziati a seconda dei destinatari per gli immigrati adulti in Italia, per i bambini e per i ragazzi figli di immigrati in Italia, per i ragazzi figli di emigranti italiani all'estero, per gli stranieri adulti che apprendono l'italiano all'estero e con tipologie linguistiche lontane dall'italiano (asiatici) e infine pergli anziani. II Centro CILS ha ricevuto dal Consiglio d'Europa, in collaborazione con il Ministero dell'Istruzione Università e Ricerca e con il Ministero del Lavoro e delle Politiche Sociali, il Label europeo 2004, riconoscimento per i prodotti più innovativi nel campo della diffusione e dell'insegnamento delle lingue, per la ricerca e la sperimentazione che ha condotto alla progettazione dei moduli certificatori per i livelli Al e A2. 
Per quanto riguarda l'italiano, il fatto che esistano più certificazioni, come del resto accade per altre lingue (inglese, francese, russo, ecc.), non deve essere letto come occasione di confusione e disorientamento, ma al contrario come fenomeno che nella molteplicità è anche garanzia di eticità, se è accompagnato da una sollecitazione alla ricerca scientifica, al dibattito e alla trasparenza delle scelte teoriche e operative adottate. Del resto anche le politiche europee tendono a sollecitare la molteplicità delle offerte formative e valutative in una prospettiva di approfondimento sulla verifica e valutazione della competenza linguistico-comunicativa basata su criteri condivisi e trasparenti. $\dot{E}$ dunque auspicabile che l'offerta certificatoria rimanga in questa dimensione di molteplicità. Solo con la molteplicità dell’offerta sarà infatti possibile dare spazio alla pluralità, al dialogo e al confronto; in caso contrario si rischierebbe di dare spazio

a operazioni meramente politiche o commerciali che non risponderebbero alle condizioni necessarie per lo sviluppo di una società democratica.

\section{Bibliografia essenziale}

Balboni P.E., 1999. Parole comuni, culture diverse. Guida alla comunicazione interculturale, Venezia, Marsilio.

Balboni P.E., 2002. Le sfide di Babele. Insegnare le lingue nelle società complesse, Torino, UTET.

Barki P., Gorelli S., Machetti S., Sergiacomo M.P., Strambi B., 2003. Valutare e certificare l'italiano di stranieri. I livelli iniziali, Perugia, Guerra Editore.

Barni M., Villarini A., (a cura di) 2001. La questione della lingua per gli immigrati stranieri: insegnare, valutare e certificare le competenze in italiano L2, Milano, Franco Angeli.

Barni M., (2005). Valutare e certificare l'italiano di stranieri: la proposta della CILS - la Certificazione di Italiano come Lingua Straniera dell'Università per Stranieri di Siena, in Jafrancesco, 2005, 40-60.

Cartura M., 2000. Insegnare l'italiano a immigrati in Dolci R., Celentin P., (a cura di), 2000. La formazione di base del docente di italiano per stranieri, Roma, Bonacci.

Council of Europe, 2001. Common European Framework of Reference for Languages: Learning, Teaching, Assessment, Council for Cultural Co-operation, Modern Languages Division, Strasbourg, Cambridge, Cambridge University Press. (Traduzione italiana a cura di Bertocchi D., Quartapelle F., 2002. Quadro Comune Europeo di Riferimento per le lingue: apprendimento, insegnamento, valutazione, Milano-Firenze, RCS Scuola-La Nuova Italia).

De Mauro T., Vedovelli M., Barni M., Miraglia L., 2002. Italiano 2000. I pubblicie le motivazioni dell'italiano diffuso fra stranieri, Roma, Bulzoni.

Gallina F., 2004. Adolescenti immigrati nella scuola superiore italiana: la questione della lingua, Tesi di laurea specialistica non pubblicata, Università per Stranieri di Siena.

Diadori P., 2001. Insegnare italiano a stranieri, Firenze, Le Monnier.

Gallina F., Alunni stranieri nelle scuole superiori: una presenza in crescita, in 'Educazione Permanente', 2005.Nuova Serie, Perugia, Guerra Edizioni.

Giacalone Ramat, A., 1986. L'apprendimento spontaneo di una seconda lingua, Bologna, Il Mulino.

Giacalone Ramat, A., 2003. Verso l'italiano, Roma, Carocci.

Jafrancesco E., (a cura di), 2005. L'acquisizione dell'italiano L2 da parte di immigrati adulti, Atti del XIII Convegno Nazionale ILSA, Roma, Edilingua.

Micheli P., 2004. Ultime tendenze negli approcci didattici, (in corso di stampa) Atti del I ${ }^{\circ}$ Simposio di Studi Linguistici dell'Italiano: Tendenze dell'Italiano Contemporaneo e Insegnamento della Lingua Italiana in Brasile - Università Federale di Rio de Janeiro, Dipartimento di Lettere Neolatine - Programma di Post-Graduaçao em Letras Neolatinas (29 marzo - 3 aprile 2004).

Tosi A., 1995. Dalla madrelingua all'italiano. Lingua e educazione linguistica nell'Italia multietnica, Firenze, La Nuova Italia.

Vedovelli M., 2000. Guida all'italiano per stranieri. La prospettiva per il Quadro comune europeo per le lingue, Carocci, Roma.

Vedovelli M., 2003. Note sulla glottodidattica italiana oggi. Problemi e prospettive, "SILTA» 2, 173-197.

Vedovelli M., Villarini A., 2003. Dalla linguistica acquisizionale alla didattica acquisizionale: le sequenze sintattiche nei materiali per l'italiano L2 destinati agli immigrati stranieri, in Giacalone Ramat, 2003.

Vedovelli M., (a cura di), 2005a. Manuale della Certificazione dell'italiano L2, Carocei, Roma.

Vedovelli M., 2005b. L'italiano nel mondo da lingua straniera a lingua identitaria: il caso "freddoccino", "SILTA" XXXIV 3, 585-609. 